\title{
New Lightning Detection Networks in Poland - LINET and LLDN
}

\author{
Marek Loboda*, Hans D. Betz, Piotr Baranski, Jan Wiszniowski and Zdzislaw Dziewit
}

Warsaw University of Technology, Koszykowa 75, 00-665 Warsaw, Poland

\begin{abstract}
Lightning detection in Poland is performed by means of a PERUN (Safir 3000) system operated by the Institute of Meteorology and Water Management. Poland is also partly covered by a VLF/LF lightning detection system (CLDN, Central Lightning Detection Network). Both sources of lightning data have their limitations resulting from detection technique, limited number of sensors and geographical configuration, with the consequence of shortcomings in the data quality. For this reason, a new network has been installed in Poland and started continuous real-time operation in May 2006. It is LINET that covers entire Poland and is complemented by numerous sensors positioned in surrounding countries. In 2007 additional LINET sensors have been installed in Poland in order to allow exploitation of reduced baselines for efficient achievement of total lightning. In the frame of the COST P18 Action "Physics of Lightning Flash and Its Effects" another new Polish project started in 2006 related to regional lightning location. At present, the Local Lightning Detection Network (LLDN) undergoes installation in the region of Warsaw. LLDN will consist of six individual stations equipped with E-field antennae and digital recorders synchronized with GPS time signals. The aim of LLDN installation is complement other networks covering region of Warsaw (PERUN, LINET) and to provide an additional source of lightning CG data with high sensitivity in a relatively small area. In the paper are described general characteristics of LINET in Poland, as well as basic characteristics and assumed performance of LLDN, which will start operation in 2008.
\end{abstract}

Keywords: Lightning detection, lightning location, cloud lightning, VHF-network, VLF/LF-network.

\section{INTRODUCTION}

Lightning location networks are operational in many countries of the world and have reached a high level of sophistication. Both cloud-to-ground (CG) and cloud (IC) lightning are reported by numerous systems that utilize mostly the low- (VLF/LF) or the high- (VHF) frequency range. Nevertheless, improvements of local networks are desirable with respect to detection efficiency, location accuracy and the capability to determine total lightning (locating of both CG and IC). Apart from technical issues, many fundamental questions of both charge separation and discharge processes remain to be clarified in atmospheric electricity. Although two different operational lightning location networks are already collecting data in Poland, two additional systems have been implemented: the first one, LINET, is an operational VLF/LF network that covers entire Poland and has already provided useful data; the second one, LLDN, is still in the set-up stage within a limited area around Warsaw and is intended to pursue specific scientific research issues. In this contribution, we scrutinize comparisons between the two larger systems, LINET and PERUN, which operate in the VLF/LF and VHF regime, respectively. It is shown that due to the combination of network data new insight can be reached. The particular example of cloud lightning is highlighted, an area that has received surprisingly little attention in the past, at least with respect to the question of what kind of cloud processes occur and can be effectively measured with the available techniques. We focus on these results that

*Address correspondence to this author at the Warsaw University of Technology Department of High Voltage Engineering and Electrical Apparatus, ul. Koszykowa 75, 00-665 Warsaw, Poland; Tel: +48608484736; Fax: +48226251914; E-mail: marek.loboda@ien.pw.edu.pl; marekloboda@wp.pl could not be obtained alone with the previously existing networks, but we do not extensively discuss other important issues such as detection efficiencies or location accuracies. The local network LLDN, presently in the installation process, is described and a number of planned experiments are outlined. Thus, the present work illustrates examples for the scientific progress from having a new large network and details the present status of network development in Poland.

\section{LINET In Poland}

During the past years the University of Munich has exploited modern technologies to develop a modern lightning location network in Germany named LINET, which offers a variety of novel features $[1,2]$. Since the German Weather Service began to use this data at the beginning of the season 2006, LINET was extended into surrounding countries, so that comprehensive observation of European thunderstorm activity became feasible. In the course of this network installation in Europe, supported by nowcast $\mathrm{GmbH}$ for operational reasons, Poland was equipped in a first stage with 5 sensors, complemented to 10 sensor sites during the second phase in 2007. An $11^{\text {th }}$ site in the North-West of Poland has been added in 2008. With this, Poland is covered well enough to allow comparisons with PERUN on a detailed basis. This undertaking enables interesting insight into various steps of the lightning discharge, because the two networks exploit different frequency ranges and, thus, are sensitive to different physical discharge processes.

\section{I-1. LINET Features}

Since advanced lightning location networks operate in many countries and are upgraded in regular intervals, a new system must exhibit marked differences and advantages in order to become meaningful. LINET relies solely on 
$\mathrm{VLF} / \mathrm{LF}$ techniques and does provide several useful features such as:

a) total lightning; almost equal efficiency for ground strokes (CG) and cloud lightning (IC);

b) high detection efficiency for both CG and IC with low discharge currents;

c) reliable technique for 3D-discrimination of CG and IC events (see Fig. (1) in a suited network geometry);

d) report of emission height for IC events;

e) optimised location accuracy;

f) simple design, fast and economic implementation with low costs for installation and operation.

As regards a) it may be noted that alternative solutions to obtain total lightning, i.e. the report of ground strokes and leader steps from cloud discharges, employ simultaneous use of both VLF/LF- and VHF techniques, resulting in well known complexity. Of course, any VLF/LF technique is unable to resolve individual source points from radio signals produced during stepped leader activity and other small-scale discharge processes occurring along a developing lightning channel. In other words, only VHF measurements may yield - depending on technical solutions for pulse processing thousands of data points per lightning event, while VLF/LF methods detect just one signal from a strong discharge process. VHF information is highly useful for research purposes and 3D-channel mapping, but since operational meteorological services do not need such detailed knowledge the data is finally condensed down, possibly to one report per leader channel. Insofar, LINET offers a relatively simple alternative to extract both CG and IC reports in a direct way with one and the same system. In particular, measurement techniques for IC and CG signals do not differ except in the last steps performed by the location algorithm in the central processing unit. Due to the different frequency regimes, VLF/LF and VHF signals arise from physically different process, a circumstance that will be elucidated below.

The claim of having almost equal efficiency for CG and IC results from the fact that both types of lightning exhibit EM signatures which are very similar in their frequencies and signal amplitudes, so that they can be measured in exactly the same way. It must be conceded, though, that IC amplitudes extend down to extremely small values, which can no longer become located within a network with baselines that are adequate for retrieving data in the entire country. However, the IC events actually located with the $\mathrm{VLF} / \mathrm{LF}$ technique are as abundant as the VHF-located IC events (see below) so that the term 'total lightning' is justified, though different parts of cloud lightning are detected.

Point b) affects detection efficiency and total lightning capability. LINET technique implies that a wealth of hitherto unmeasured low-amplitude events become located. Incidentally, 'event' is defined as a VLF/LF waveform well separated in time by other similar signals of this kind; thus, in the frequency range considered, an event represents either a CGor an 'IC-stroke' (see term definition below). Although it was generally known that characteristic lightning IC events radiate in the VLF/LF range specific exploitation did not take place in the past. We find that sensor baselines of the order of $\sim 200 \mathrm{~km}$ allow efficient detection of relatively weak IC-strokes with currents below $\sim 5 \mathrm{kA}$. Among the advantages of recording low-amplitude events it may be mentioned that the large number of detected strokes enhances application of cell tracking, especially with respect to early recognition of severe weather conditions.

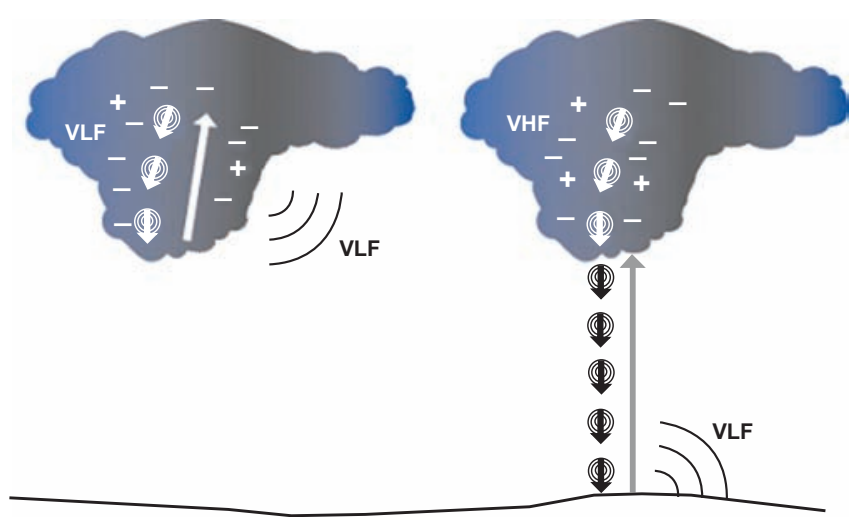

Fig. (1). Scheme for the emission of VLF/LF and VHF radiation during a lightning discharge. CG strokes are readily detected by VLF/LF techniques; in principle, the stepped leader channel connecting to ground could be identified from VHF radiation (in a 3D network). Likewise, cloud lightning produces leader steps that can be located by VHF systems. Less well known is the fact that cloud discharges typically emit also in the VLF/LF regime: relatively strong IC-strokes can be located by means of the LINET technology. The shown charges are not intended to reflect real charge structures; the arrows indicate waves in lightning channels that produce EM radiation.

It is fair to add that the quantification of IC currents as 'CG-equivalent current' may be erroneous. We apply the range normalization exactly as for CG strokes, but a rigorous justification is not at hand. The normalization procedure relies on the assumption that charge acceleration and wave speed in the lightning channel are similar for CG and IC. A more reliable procedure cannot be applied until the mechanism of IC discharges is better understood. In any case, when field amplitudes instead of currents are considered, the described similarities between CG and IC signal features remain valid.

Features c) and d) are less influential for most meteorological applications of lightning data, but c) is important for assessment of damage on the ground. Conventional VLF/LFmethods for CG-IC discrimination suffer from widely published shortcomings while the LINET 3D-technique seems to be more reliable. Its applicability is ensured as long as the sensor baselines in the network are short enough and do not exceed some $200-250 \mathrm{~km}$, and certain site error corrections are applied. According to point e), LINET provides improved statistical location accuracy and almost excludes the occurrence of erroneous locations. The latter feature is particularly important for determination and control of damage by CG strokes. More details about LINET can be found in $[3,4]$.

The presently adopted term "IC-stroke" is not customary, so that it seems adequate to give a brief explanation. Traditionally, automated networks exploit the VLF/LF and VHF ranges with centre frequencies around $10 \mathrm{kHz}$ and $100 \mathrm{MHz}$ for CG and IC detection, respectively. Cloud lightning pro- 
duces signatures in both ranges, but the VLF/LF regime has not been utilized for locating with networks. More specifically, networks for CG reporting have taken specific measures to remove IC contributions from the records and often mix CG-IC type classification. Since the IC signals appear in large numbers and show waveforms and amplitudes not too different from what is observed for CG strokes, the term "IC-stroke" appears justified, but is not intended to suggest any explanation about its production mechanism.

\section{I-2. Verification of LINET Data}

During the past years the quoted LINET features have been thoroughly controlled and verified. While high-current strokes can be reliably detected in all networks, low-current events are more difficult to locate. As a matter of fact, lower detection thresholds are often not specified or discussed in the literature and true low-amplitude distributions have never been presented. Therefore, uncertainty remains with respect to the definition of the $100 \%$-level for CG stroke-detection efficiency. The problem is heavily amplified when it comes to cloud lightning, where no information is available about the very small amplitudes of individual IC-strokes. In many experiments it was found that CG strokes are abundant below $10 \mathrm{kA}$, where IC-components become dominant and most networks begin to exhibit deteriorating detection efficiency. Reduction of sensor baselines would remedy such shortcomings only to some extent, because the chosen procedures for measurement and handling of individual small signals are extremely influential.

In Southern Germany, LINET data was intensively checked for many years by comparison with BLIDS and ALDIS results, thanks to scientific co-operations with DWD (German Weather Service), Siemens and ALDIS (Austrian Lightning Detection and Information System); some characteristic comparisons have been published [5]. In addition, DWD scrutinized LINET events using a variety of meteorological data sources, including lightning data from BLIDS and radar images. Finally, the IPA Institute of DLR (Institute for Physics of the Atmosphere, Deutsches Zentrum für Luftund Raumfahrt) was equipped with a 7-sensor LINET version for utilization in international field campaigns. The first project was carried out in Brazil (region around Bauru), others followed in Australia (Darwin area) and Africa (mainly Benin). In Brazil, LINET could be compared with the local national lightning detection network (RINDAT), proving the same advantages of LINET as observed in Germany [6]. Since LINET data is available in at least 16 European countries and receives increasing attention in many scientific programs, data quality control can be performed on a broad basis. The upcoming comparisons with LLDN in the Warsaw area represent one of the attempts for future data verification.

\section{I-3. LINET Configuration in Poland}

The first LINET sensors in Poland were installed in Warsaw, Gdańsk, Białystok, Rzeszów and Poznań in 2006. Since the baselines were large, the network was extended by installing six additional sensors. The configuration of LINET in Poland with ten sensors as of 2007 is shown in Fig. (2). Each sensor is equipped with magnetic crossed-loop antenna and a GPS module, connected to a field processor (Fig. 3). Each site has a permanent connection with the internet and is remotely controlled. The data collected from each individual station are transmitted to a central analyser unit in Munich where the final processing is carried out. The data set for each individual lightning stroke contains time of the event, type (IC and CG with quality assigmant), polarity, rangenormalized current amplitude, location error, participating sensors, digitised wave-forms, and a variety of other control parameters. It is also possible to access this information via internet and to visualize and animate archived events.

\section{I-4. Examples of Comparison of LINET and PERUN Data}

A first comparison of lightning data obtained from the two networks PERUN (see system description in [7]) and LINET has been presented for storms in the season 2006 [8]. In the present contribution, we extend the comparison to a more fundamental question, namely the investigation of cloud lightning detected by LINET. It has been assumed all along that a large fraction of located strokes must originate from IC processes, but it was not obvious from where and during which parts of a discharge the relatively strong $\mathrm{VLF} / \mathrm{LF}$ signatures are produced. In a recent paper, the time coincidences between signals from VLF/LF and VHF networks have been examined $[3,9]$, with most remarkable results. As a typical example, we reproduce in Table 1 the listing of all located signals from the first five flashes observed during the storm occurring in the South-West of Poland on October 5, 2007. Attention should be given to the time sequence of reported VHF and VLF/LF signals, because this observation illuminates the crucial findings.

It turns out that the appearance of a first IC source point signal that is detected by PERUN for the flashes \#1, \#2, \#4, and \#5 is accompanied by an IC-stroke located by LINET. The cloud activity of flash \#3 is not recorded by PERUN. When the entire storm is analysed, one finds time-coincident LINET-strokes for up to about $50 \%$ of the first PERUN ICevents. There are also PERUN reports without matching LINET strokes and vice versa, not expanded here. This finding is in qualitative agreement with many long-standing reports in the literature; in the past, however, the large fraction of time coincidences has not been realized under quantitative aspects. A detailed discussion of the underlying processes is beyond the scope of this contribution, but must be regarded quite obviously as a step forward towards understanding of cloud lightning [9]. As a consequence, it is now more transparent that LINET is capable of reporting cloud events and, thus, total lightning very efficiently: cloud lightning is typically accompanied by relatively strong strokes that arise during the initial breakdown, surprisingly with no measured preceding stepped-leader activity, and can be measured in the VLF/LF regime. This implies the remarkable circumstance that VLF/LF techniques are capable of reporting cloud activity (total lightning) without making use of VHF components. Fig. (4) proves that comprehensive detection is possible; in fact, for the present storm LINET reports more strokes than PERUN locates leader channels.

In Fig. (5) the distribution of those range-normalized LINET currents is shown that have occurred in close timecoincidence with a first IC-source point located by PERUN. It becomes obvious that the IC-strokes are only slightly weaker than the ones typically found for CG strokes; in any case, the two distributions overlap significantly. Note for example, that the first IC-stroke in flash \#4 exhibits a current 


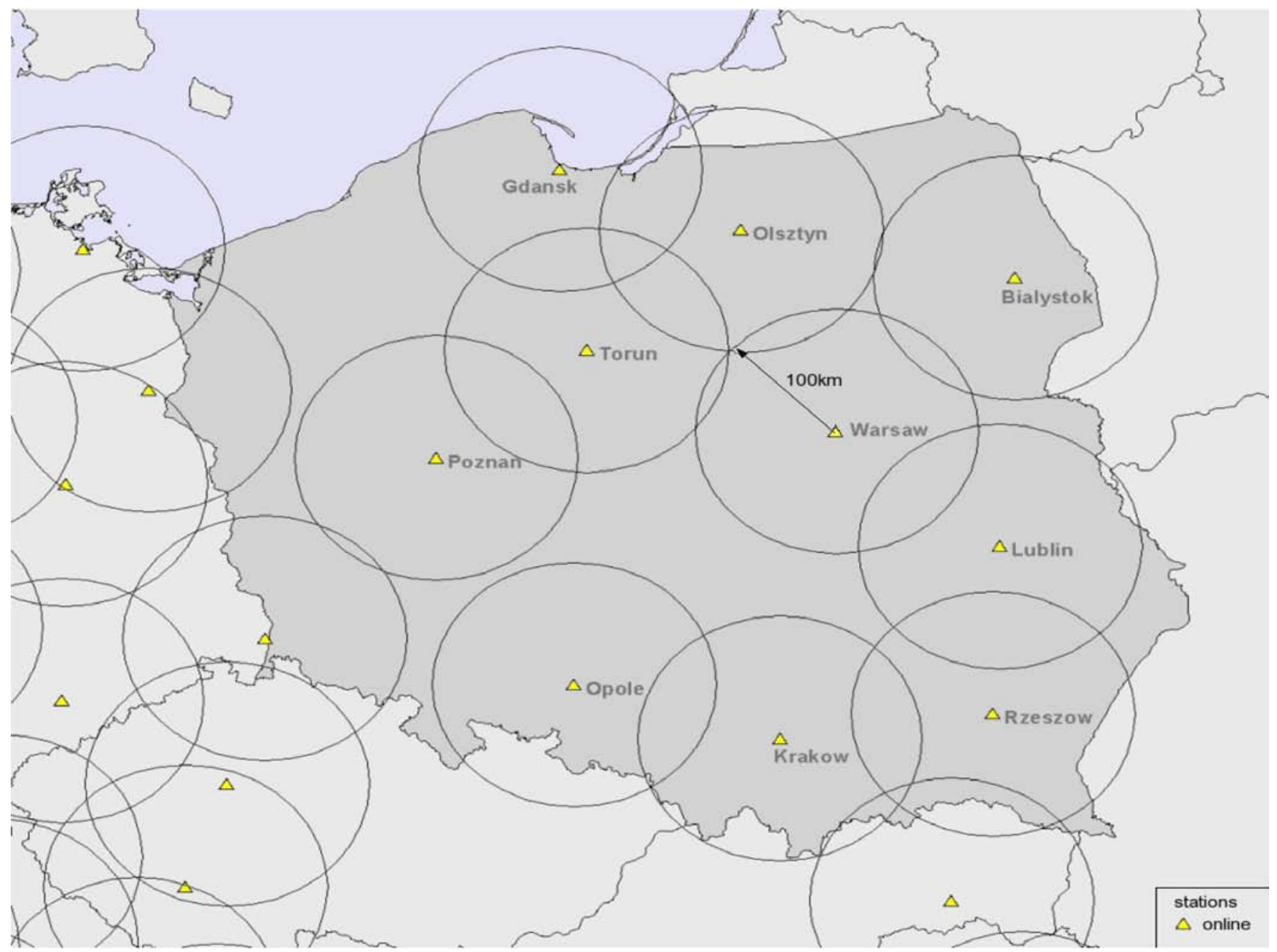

Fig. (2). Location of LINET sensors in Poland; circles with $100 \mathrm{~km}$ radius serve to guide the eye and indicate areas with reliable IC-CG discrimination. When the distance between lightning and the nearest reporting sensor becomes larger, uncertainties may arise in the discrimination. The location accuracy is less affected by the sensor baseline, because time-of-arrival is employed and direction-finding serves as verification of locations.

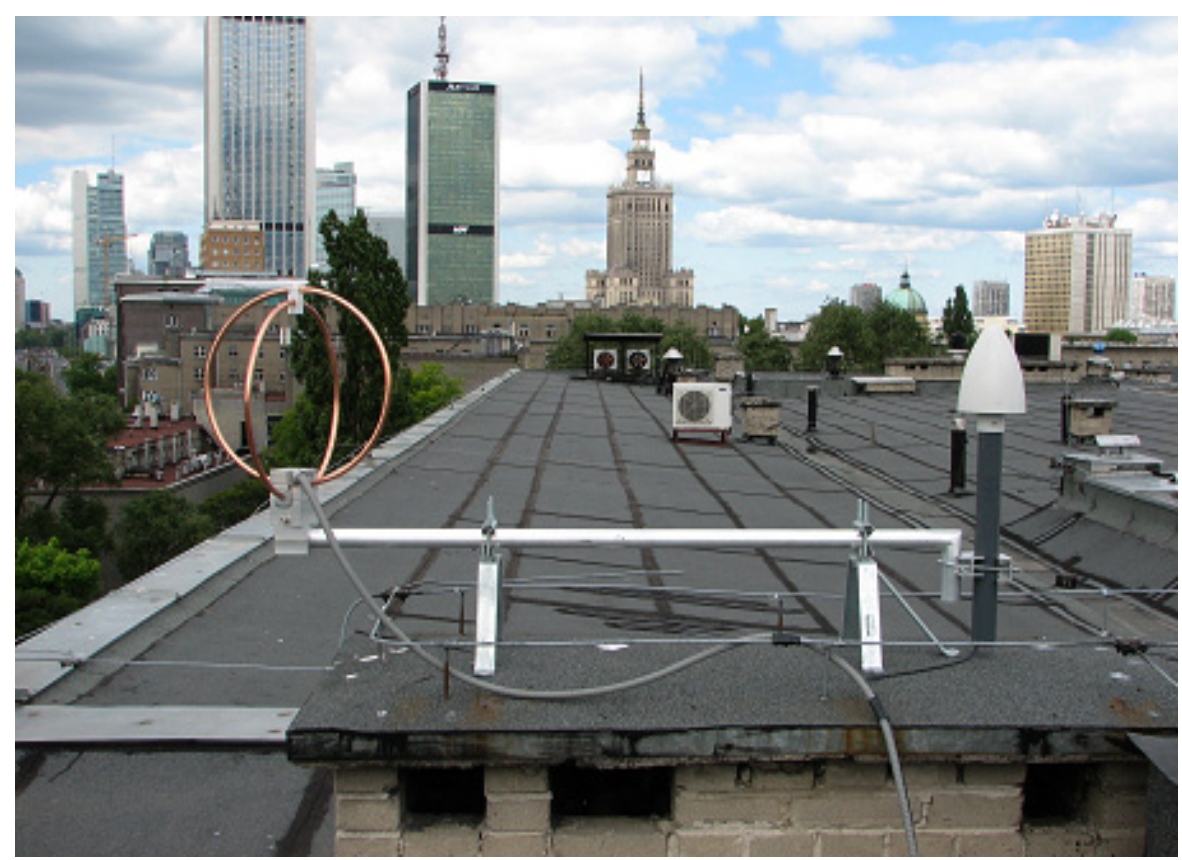

Fig. (3). View of LINET sensor set-up in Warsaw with field antenna (two crossed loops) and GPS antenna. 
Table 1. The First Five Flashes of October 5, 2007, as Detected by PERUN and LINET. Columns 1, 2, 6, 7: Event Time; 3: Source Type (1=Start, 2=Intermediate, 3=End of IC Source Train; 4=CG); 4, 11: Stroke Current; 8, 9: 2D-Location; 10: Source Height. The Stroke Currents are Range-Normalized Field Amplitudes and are Given Only for VLF/LF Pulses

\begin{tabular}{|c|c|c|c|c|c|c|c|c|c|c|}
\hline 1 & 2 & 3 & 4 & 5 & 6 & 7 & 8 & 9 & 10 & 11 \\
\hline \multicolumn{4}{|c|}{ PERUN } & \multirow[b]{2}{*}{ flash } & \multicolumn{6}{|c|}{ LINET } \\
\hline hh:mm:ss & ms & type & kA (CG) & & hh:mm:ss & ms & lon & lat & km (IC) & $\mathrm{kA}(\mathrm{IC}, \mathrm{CG})$ \\
\hline 01:07:30 & 493,2 & 1 & & $\# 1$ & 01:07:30 & 493,2 & 16,0448 & 52,9532 & 9,8 & $-8,6$ \\
\hline 01:07:30 & 493,3 & 2 & & & & & & & & \\
\hline 01:07:30 & 493,4 & 2 & & & & & & & & \\
\hline 01:07:30 & 493,5 & 2 & & & & & & & & \\
\hline 01:07:30 & 493,6 & 2 & & & & & & & & \\
\hline 01:07:30 & 493,8 & 3 & & & & & & & & \\
\hline \multirow[t]{3}{*}{ 01:07:30 } & \multirow[t]{3}{*}{501,0} & \multirow[t]{3}{*}{4} & \multirow{3}{*}{$-20,6$} & & 01:07:30 & 501,0 & 16,0480 & 52,9690 & 0 & $-22,1$ \\
\hline & & & & & 01:07:30 & 509,7 & 16,0489 & 52,9591 & 0 & $-8,5$ \\
\hline & & & & & 01:07:30 & 527,2 & 16,0458 & 52,9519 & 8,0 & 9,1 \\
\hline 01:08:47 & 457,1 & 1 & \multirow{7}{*}{$-9,7$} & \multirow[t]{7}{*}{$\# 2$} & \multirow[t]{5}{*}{ 01:08:47 } & \multirow[t]{5}{*}{457,1} & \multirow[t]{5}{*}{16,0497} & \multirow[t]{5}{*}{52,9485} & \multirow[t]{5}{*}{8,1} & \multirow[t]{5}{*}{$-6,0$} \\
\hline 01:08:47 & 457,2 & 2 & & & & & & & & \\
\hline 01:08:47 & 457,4 & 2 & & & & & & & & \\
\hline 01:08:47 & 457,5 & 2 & & & & & & & & \\
\hline 01:08:47 & 457,6 & 3 & & & & & & & & \\
\hline \multirow[t]{2}{*}{ 01:08:47 } & \multirow[t]{2}{*}{473,7} & \multirow[t]{2}{*}{4} & & & 01:08:47 & 473,7 & 16,0586 & 52,9517 & 7,3 & $-10,3$ \\
\hline & & & & & 01:08:47 & 487,0 & 16,0403 & 52,9463 & 9,5 & $-6,0$ \\
\hline \multirow{7}{*}{ 01:09:49 } & \multirow{7}{*}{475,6} & \multirow{7}{*}{4} & \multirow{7}{*}{$-16,1$} & $\# 3$ & 01:09:49 & 397,5 & 16,0593 & 52,9560 & 8,0 & $-19,1$ \\
\hline & & & & & 01:09:49 & 429,2 & 16,0726 & 52,9676 & 8,7 & $-11,8$ \\
\hline & & & & & 01:09:49 & 475,6 & 16,0360 & 52,9460 & 8,1 & $-16,1$ \\
\hline & & & & & 01:09:49 & 505,6 & 16,0517 & 52,9554 & 7,7 & $-13,7$ \\
\hline & & & & & 01:09:49 & 555,2 & 16,0361 & 52,9460 & 8,9 & $-10,9$ \\
\hline & & & & & 01:09:49 & 739,7 & 16,0364 & 52,9460 & 9,7 & $-6,8$ \\
\hline & & & & & 01:09:49 & 788,8 & 16,0362 & 52,9459 & 9,6 & $-7,4$ \\
\hline 01:11:38 & 595,5 & 1 & & $\# 4$ & 01:11:38 & 595,5 & 16,2400 & 53,0674 & 9,7 & 65,6 \\
\hline $01: 11: 38$ & 595,6 & 2 & & & & & & & & \\
\hline 01:11:38 & 595,7 & 2 & & & & & & & & \\
\hline 01:11:38 & 595,8 & 2 & & & & & & & & \\
\hline & & & & & 01:11:38 & 605,6 & 16,2363 & 53,0652 & 0,0 & $-6,9$ \\
\hline & & & & & 01:11:38 & 631,7 & 16,2079 & 53,0590 & 0,0 & $-10,7$ \\
\hline & & & & & $01: 11: 38$ & 665,6 & 16,2193 & 53,0740 & 0,0 & 12,6 \\
\hline & & & & & 01:11:38 & 687,4 & 16,2185 & 53,0744 & 9,1 & 12,9 \\
\hline & & & & & 01:11:38 & 689,0 & 16,1290 & 53,0568 & 0,0 & 4,1 \\
\hline 01:11:38 & 789 & 4 & $-8,0$ & & 01:11:38 & 789,0 & 16,2197 & 53,0758 & 0,0 & $-8,1$ \\
\hline & & & & & 01:11:38 & 818,7 & 16,2196 & 53,0742 & 0,0 & $-8,9$ \\
\hline $03: 29: 26$ & 379,7 & 1 & & \# 5 & $03: 29: 25$ & 990,5 & 15,0041 & 50,5977 & 0 & $-8,6$ \\
\hline $03: 29: 26$ & 379,8 & 2 & & & $03: 29: 26$ & 379,6 & 15,3268 & 50,6558 & 9,5 & 29,2 \\
\hline $03: 29: 26$ & 379,9 & 2 & & & & & & & & \\
\hline $03: 29: 26$ & 380,0 & 3 & & & & & & & & \\
\hline $03: 29: 26$ & 382,4 & 1 & & & & & & & & \\
\hline $03: 29: 26$ & 382,7 & 2 & & & & & & & & \\
\hline $03: 29: 26$ & 382,9 & 3 & & & & & & & & \\
\hline
\end{tabular}




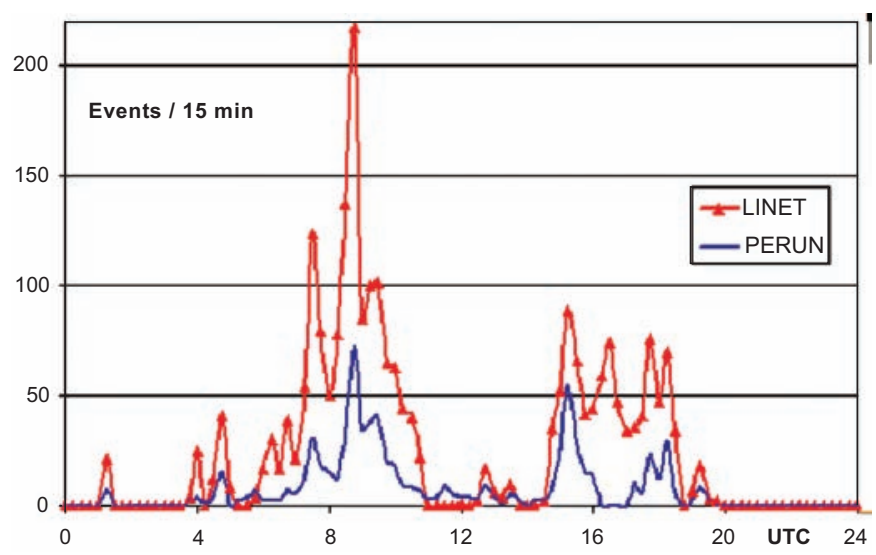

Fig. (4). Number of IC leader-channels and IC-strokes located by PERUN and LINET, respectively, as a function of time (bin size 15 min), for the storm on Oct. 5, 2007 (taken from [4]).

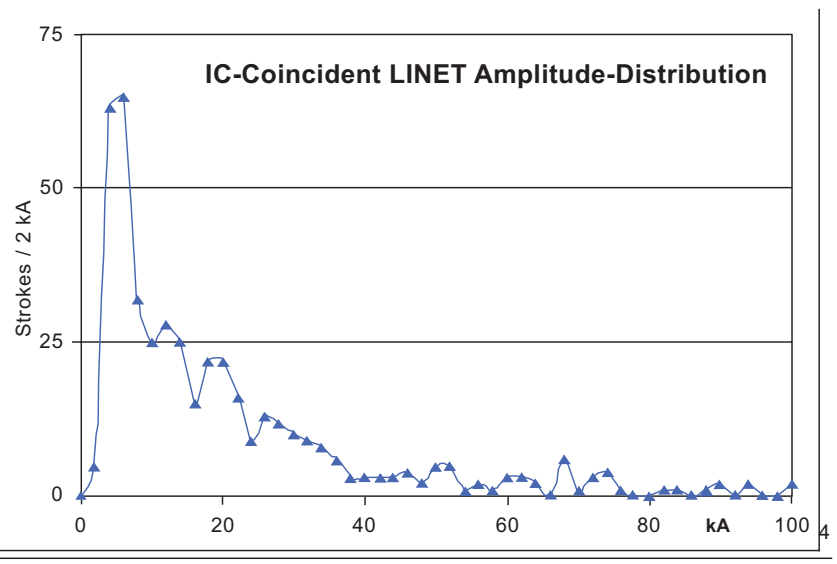

Fig. (5). Amplitude distribution of LINET IC-strokes, timecoincident with first IC source points reported by PERUN for stepped leader channels.

of $65 \mathrm{kA}$. The entire data set comprises currents well beyond $100 \mathrm{kA}$. The 2D-locations of the coincident signals are shown in Fig. (6): there is a large discrepancy between the two network reports. Fig. (7) displays the distribution of location differences. Obviously, the PERUN IC location accuracy is far from the expected values, probably a result of the unfavourable location in a border region of the network and the use of intrinsically imprecise direction finding. For coincident CG strokes the agreement is significantly better.

In the central part of Poland the two networks show smaller discrepancies between time-coincident IC locations. Nevertheless, careful investigation of many other storms, along with comparisons between LINET and SAFIR-type systems in three different countries confirm that the used VHF networks produce IC locations with quite large errors. Although precise IC locations are far less important than CG strike points, storm cell recognition is much more useful when reliable locations are provided; more comprehensive statistical evaluations will be presented elsewhere.

As a result of the data comparisons a number of conclusions can be stated. PERUN provides a representative picture for cloud lightning, though the number of located channels per flash remains limited. The 2D-location of the VHF source points deviate significantly from the locations of time-coincident LINET IC-strokes; provided that the
VLF/LF signals are emitted from the VHF-located channels, much better agreement should be expected. The locations of CG strokes from PERUN and LINET agree very well, though PERUN reports CG strokes with high efficiency only for currents well above some $15 \mathrm{kA}$. The two networks detect total lightning with quite similar efficiency; this must be considered as a novelty, because hitherto VLF/LF systems have not been considered as a tool that can be effective in the localization of cloud activity. Comparisons will be continued in the forthcoming season, including stroke-by-stroke analysis of data from the upcoming LLDN.

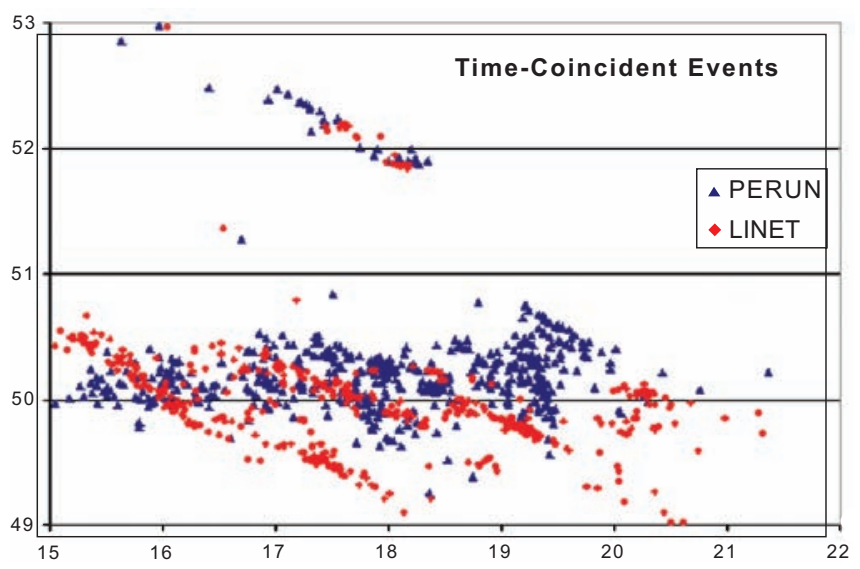

Fig. (6). Lightning map with the locations of time-coincident signals from the two networks (latitude and longitude in deg).

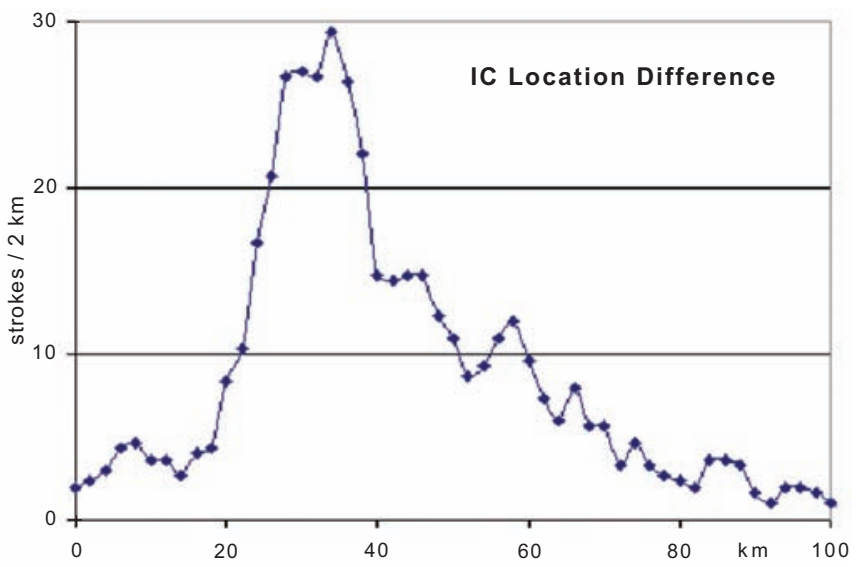

Fig. (7). Location difference between the IC source points and time-coincident IC-strokes from Fig. 6 (bin size $2 \mathrm{~km}$ ).

\section{LLDN - Local Lightning Detection Network In War- saw}

As a result of the European cooperation in the frame of COST P18 Action "Physics of Lightning Flash and Its Effects", in March 2007 started the new project related to the installation of a Local Lightning Detection Network LLDN in the region of Warsaw. This project is a result of cooperation between three Polish institutions - Warsaw University of Technology, Institute of Geophysics in Warsaw, and Institute of Meteorology and Water Management. The main goals of this project are:

creation of new network LLDN, which is intended to complement other networks and to provide an addi- 
tional source of lightning CG data with high sensitivity in a relatively small area;

to compare LLDN data for individual events with those recorded by the Polish PERUN (SAFIR) system operated by the Institute of Meteorology and Water Management in Warsaw, and by LINET;

- to derive physical characteristics of individual CG strokes from lightning data recorded by LLDN, such as locations of strokes, current values of individual lightning strokes, and characteristics of multiple stroke discharges.

\section{II-1. Configuration and Features of LLDN}

The LLDN will consist of six measuring stations installed in Warsaw are creating network configuration as shown in Fig. (8). The planned space configuration of LLDN in Warsaw will based on one reference E-field measuring point, installed in open area in site having flat surroundings (Bemowo). This antenna will be used for comparison of E-field parameters measured by each station. The stations will be located in distance not longer than of $10 \mathrm{~km}$ between each one, what enables to record simultaneously by each station E-field signals of the same of it polarity generated by close CG lightning discharges (inside of so-called reversal distance).

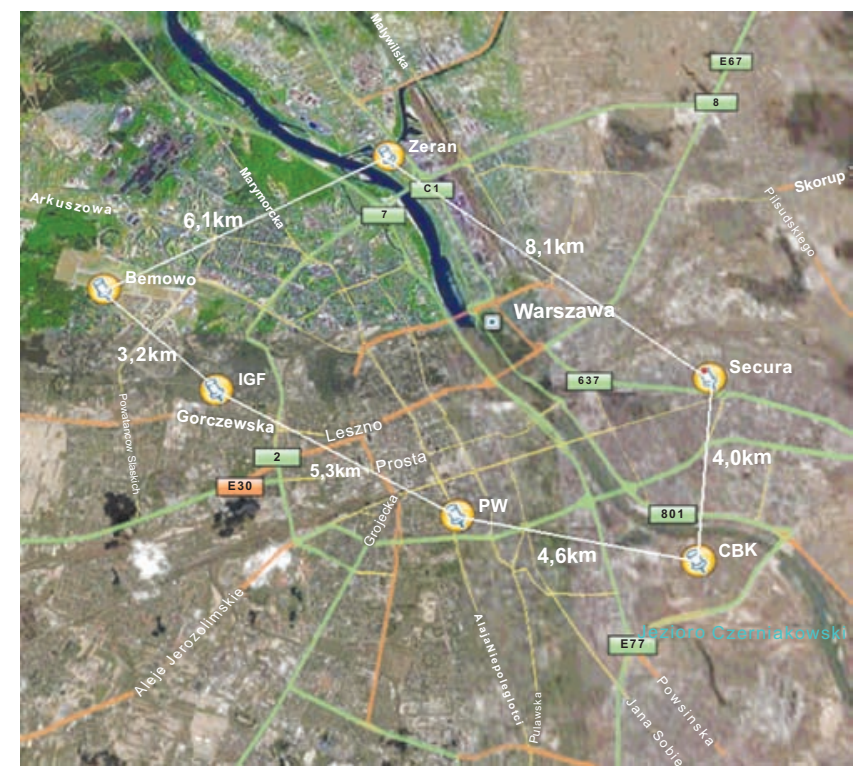

Fig. (8). Planned locations of six sensors and recorders of LLDN in
Warsaw with estimated distances between individual stations.

Each station will consist of:

- two electric field antennae (flat and rod) utilizing the frequency band from $20 \mathrm{~Hz}-20 \mathrm{kHz}$, with an implemented electric field amplifier having an input gain $>60 \mathrm{~dB}$ and a time constant $1 \mathrm{~s}$, and a pre-trigger 14bit $\mathrm{A} / \mathrm{D}$ converter with individual memory storage bank; the aim of the rod antenna installation is to calibrate amplitude of electric field sensors using selected radio broadcast signal $(225 \mathrm{kHz})$;

- two-channel digital data recorder;

- GPS antenna used for time synchronization of individual stations.

An additional LF calibrated reference antenna will be installed in one of sites at the earth surface.

In addition, two high-speed cameras and one field mill will be used during the measurement campaign throughout the lightning season 2008. Some fundamental characteristics of the station equipment are given in Table 2, and Fig. (9) shows a block diagram of the individual measuring stations and the recording system.

\section{II-2. Methodology of Lightning Data Analysis Recorded by LLDN}

Table 3 lists the geographic coordinates for the individual stations of the LLDN (measuring points, columns 2 and 3). In addition, columns 5 and 6 shown the same points with their location assignments computed according to the local Cartesian coordinate set that will be used for the purpose of future data evaluation. These coordinates are converted according to the WGS-84 [10]. The altitude of a particular station, $h$, as well as the Elevation will be omitted in the calculations below, because in the considered small area the values do not change much from site to site. Measured signals are expected to arise from multiple cloud-to-ground lightning flashes, comprising stepped leaders, return strokes, or continuing currents, and are intended to be used for the determination of charge values. Of course, some prominent cloud strokes will also be present in the recordings and must be treated adequately.

The change of the electrostatic electric field recorded at the ground and caused by a lightning discharge of a certain thundercloud electric charge volume can be obtained at the $i$ th station from the point charge relation [11]:

Table 2. Fundamental Characteristics of LLDN Station Equipment

\begin{tabular}{|c|c|c|c|}
\hline \multicolumn{2}{|c|}{ E - Field Meter with AC Component } & \multicolumn{2}{c|}{ Two Channel Data Recorder } \\
\hline Parameter & Value & Parameter & Value \\
\hline \hline E- field strength & up to $\pm 20 \mathrm{kV} / \mathrm{m}$ & $\begin{array}{c}\text { Frequency range: Slow mode } \\
\text { Fast mode }\end{array}$ & $\begin{array}{c}20.0[\mathrm{~Hz}] \text { to } 20.0 \\
{[\mathrm{kHz}] 20.0[\mathrm{~Hz}] \text { to } 100.0} \\
{[\mathrm{kHz}]}\end{array}$ \\
\hline Estimated distance to stroke location & $1 \mathrm{~km} \div 12 \mathrm{~km}$ & $\begin{array}{c}\text { Sampling rate: Slow mode } \\
\text { Fast mode }\end{array}$ & $\begin{array}{c}50.0[\mathrm{kS} / \mathrm{s}](20.0 \mathrm{~ms}) \\
250.0[\mathrm{kS} / \mathrm{s}](4.0 \mathrm{~ms})\end{array}$ \\
\hline Error for $\Delta \mathrm{E}_{\mathrm{min}}$ & $<1 \% /:+20 \mathrm{~dB}$ & Dynamic range & $80[\mathrm{~dB}]$ \\
\hline Range ability (gain difference between low and high range): & $>85 \mathrm{~dB}$ & $\begin{array}{c}\text { One shot recording time } \\
\text { Pre-triggering time }\end{array}$ & $2 \times 1.0[\mathrm{~s}]$ \\
$0.5[\mathrm{~s}]$
\end{tabular}




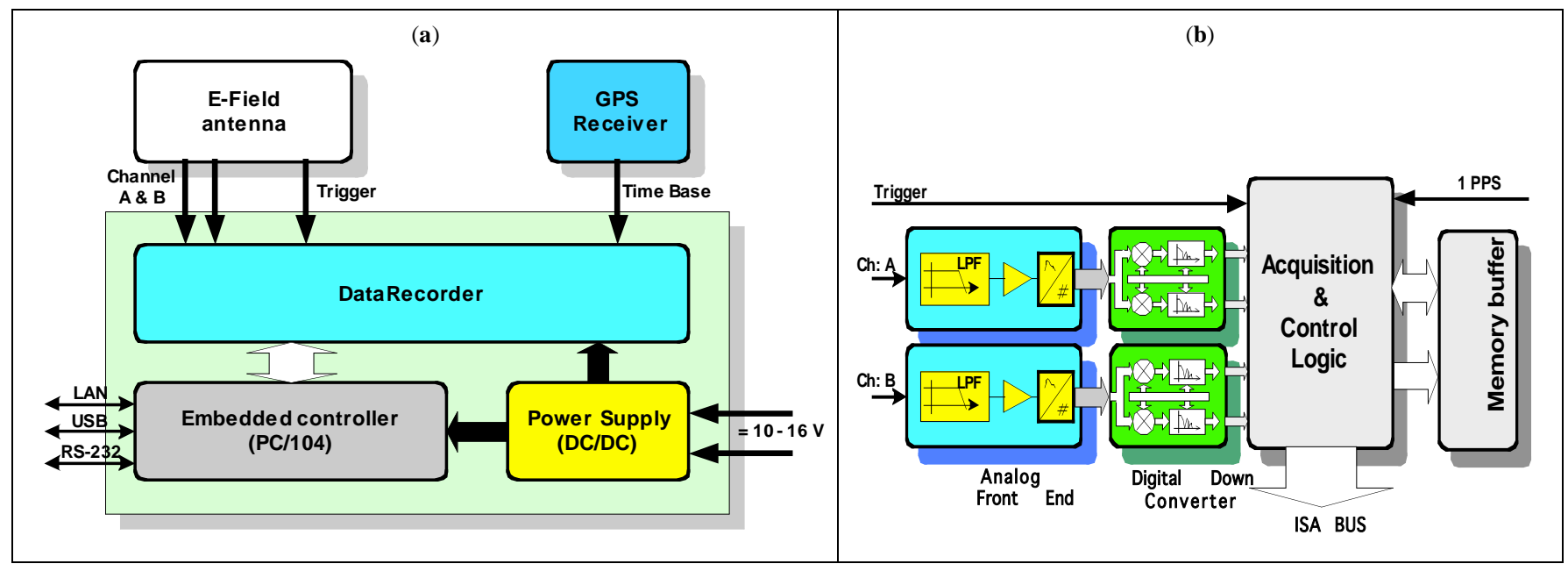

Fig. (9). Block- diagrams of measuring station (a) and recorder (b).

$$
\Delta E_{i}=\frac{1}{4 \pi \varepsilon_{0}} \frac{2 \Delta Q z}{\left[\left(x-x_{i}\right)^{2}+\left(y-y_{i}\right)^{2}+z^{2}\right]^{3 / 2}}
$$

where $\varepsilon_{o}$ is the permittivity of free space and $x, y, z$ are the coordinates of the charge centre, and $\Delta Q$ is the charge neutralized by lightning flash.

The charge parameters, i.e. $\mathrm{x}, \mathrm{y}, \mathrm{z}$ and $\Delta \mathrm{Q}$ are estimated by minimising the $\chi^{2}$ function described by the formula [12]:

$\chi^{2}=\sum_{i} \frac{\left[\Delta E_{i}-\Delta E_{i}(x, y, z, \Delta Q)\right]^{2}}{\sigma_{i}^{2}}$

The denominator $\sigma_{i}{ }^{2}$ can be omitted because it is assumed we have the same error values for all stations. The charge location and magnitude is estimated by searching a set of $\mathrm{x}, \mathrm{y}, \mathrm{z}$ and $\Delta \mathrm{Q}$ values, which is minimising the value calculated from Eq. (2). If the value of $\chi^{2}$ is consistent with the measurement error, then the used parameters are regarded as a solution. However, the function expressed in Eq. (2) is not monotonic and all minimising algorithms include gradient methods that can let to a local minimum, which cannot represent the finally searched solution. Thus, Jacobson and Krider [12], suggested employing the non-linear gradient algorithm of Marquardt. On the other hand in [11] was considered the simplex method as the better one. However, it may sometimes occur that even simulations of the computing procedures (with numerical recipes prepared in $\mathrm{C}++$ language and based on the simplex algorithm, or using the Levenburg-Marquardt method), when applied to the net configuration of measuring stations shown in Fig. (10), can lead to the wrong results. Here, it is worth to note that some limitation of the allowed range of the considered lightning parameter values, as well as to the accepted changes during the optimising procedure may reduce the number of wrong results, but will not eliminate all of them.

It was proposed [11] to compute lightning parameters by solving the set of four equations obtained for every combination of four stations from the total number of used stations, provided that electric field changes from lightning discharges are measured correctly. Any solution for $\mathrm{x}, \mathrm{y}, \mathrm{z}$ and $\Delta \mathrm{Q}$ can result from a set of three linear equations expressed as follows:

$$
2\left(x_{i}-x_{j}\right) x+2\left(y_{i}-y_{j}\right) y+\left(\Delta{E_{i}^{\prime-2 / 3}}^{-\Delta E_{j}^{\prime-2 / 3}}\right) \eta=r_{i}^{2}-r_{j}^{2}
$$

where $\eta=(2 \Delta Q z)^{2 / 3}, \Delta E_{i}^{\prime}=4 \pi \varepsilon_{0} \Delta E_{i}$ and $r_{i}^{2}=x_{i}^{2}+y_{i}^{2}$ with $i, j=1,4$ and $i \neq j$.

Table 3. Geographic Coordinates of LLDN Stations in Warsaw Region with the Assumed Base Point of the Local Cartesian Coor-

\begin{tabular}{|c|c|c|c|c|c|c|}
\hline Name of Station & Latitude $\left[{ }^{\circ}\right]$ & Longitude $\left[^{\circ}\right]$ & Elevation $[\mathrm{m}]$ & $x[\mathrm{~m}]$ & $y[\mathrm{~m}]$ & $h[\mathrm{~m}]$ \\
\hline Bemowo & $20^{\circ} 54^{\prime} 21.0^{\prime \prime}$ & $52^{\circ} 16^{\prime} 01.0^{\prime \prime}$ & 0.0 & 6964.60 & 647.29 & -3.83 \\
\hline IGF & $20^{\circ} 56^{\prime} 24.0^{\prime \prime}$ & $52^{\circ} 14^{\prime} 42.0^{\prime \prime}$ & 0.0 & 4680.53 & 4429.29 & -3.26 \\
\hline PW & $21^{\circ} 00^{\prime} 25.0^{\prime \prime}$ & $52^{\circ} 13^{\prime} 20.0^{\prime \prime}$ & 0.0 & 2310.35 & 11840.66 & -11.47 \\
\hline CBK & $21^{\circ} 04^{\prime} 04.0^{\prime \prime}$ & $52^{\circ} 12^{\prime} 53.0^{\prime \prime}$ & 0.0 & 1529.98 & 18575.87 & -27.38 \\
\hline Secura & $21^{\circ} 04^{\prime} 53.0^{\prime \prime}$ & $52^{\circ} 14^{\prime} 54.0^{\prime \prime}$ & 0.0 & 5022.51 & 20083.54 & -33.77 \\
\hline Żerań & $20^{\circ} 59^{\prime} 30.0^{\prime \prime}$ & $52^{\circ} 17^{\prime} 43.0^{\prime \prime}$ & 0.0 & 9906.61 & 10151.93 & -15.81 \\
\hline \multicolumn{7}{|c|}{ Base Point } \\
\hline Left - bottom & $20^{\circ} 54^{\prime} 00.0^{\prime \prime}$ & $52^{\circ} 12^{\prime} 00.0^{\prime \prime}$ & 0.0 & 0.0 & 0.0 & 0.0 \\
\hline
\end{tabular}
dinates 
Based on the calculated values of $x, y$ and $\eta$, we can estimate $z$ and $\Delta Q$ values according to formulas:

$z=\sqrt{\frac{\eta}{\Delta E_{i}^{\prime 2 / 3}}-\left(x-x_{i}\right)^{2}-\left(y-y_{i}\right)^{2}}$

and

$\Delta Q=\eta^{3 / 2} / 2 z$

However, when some measurement error of the electric field changes is introduced for each station of the considered four-point net, or when the electric charge involved in lightning discharge is not taken as the point source, evaluated solutions for the determined variable $z$ may assume a complex value. Nevertheless, additional numerical simulation has shown that for many possible other cases the evaluated solutions are not characterized by significant errors. Therefore, one can conclude that the proposed estimation methods for finding proper lightning parameters is essentially correct. Here, the additional assumption is made that the station location coordinates $x_{i}, y_{i}$ are not near a singularity of the searching solution, and that the electric charge is located over or near the considered network of stations. Then, the averaged solution of all combinations for each four of stations will be a good estimate of true electric charge 2D location and its magnitude. Although the number of these combinations grows according to the relation $N ! /(N-4) ! 4$ ! and their relevant solutions are not independent, the obtained estimates of true solutions may be seen as less reliable than the previous one and less accurate than that ones resulting from direct minimizing of Eq. (2). For example, when the calculation of lightning parameters is based on electric field changes recorded by each of the four stations, and when the recording contains some noise, then it may occur that noisy recordings lead to additional solutions that have a big influence on the final result. On the other hand, when lightning location is computed from four stations that are oriented along one line, one can expect a large error that will be also disturb the final result. For the case of four stations, i.e. the sites Bemowo, $I G F, P W$ and $C B K$ that are almost located along one line, it will be better to consider the final result as a weighted sum of all solutions obtained for these four stations.

An alternative way for finding lightning parameters is to minimize Eq. (2) by the global minimization algorithm. For this purpose we can also use a Monte Carlo optimisation method. Moreover, two other methods may be applied in future considerations, i.e. the simulated annealing and Metropolis-Hastings algorithm [13]. Fig. (10) presents examples of simulated 2D lightning locations for real configurations of the LLDN, obtained by the use of different estimation methods, such as the simplex algorithm and simple solving of the set of four algebraic equations.

In case (a) the simplex method was used that gives the possibility to find the global minimum; location errors from the simplex method were: $\delta \mathrm{x}=14 \mathrm{~m}$ and $\delta \mathrm{y}=8 \mathrm{~m}$, whereas those errors indicated by using the method of linear equations were $\delta x=-174 \mathrm{~m}$ and $\delta y=-127 \mathrm{~m}$.
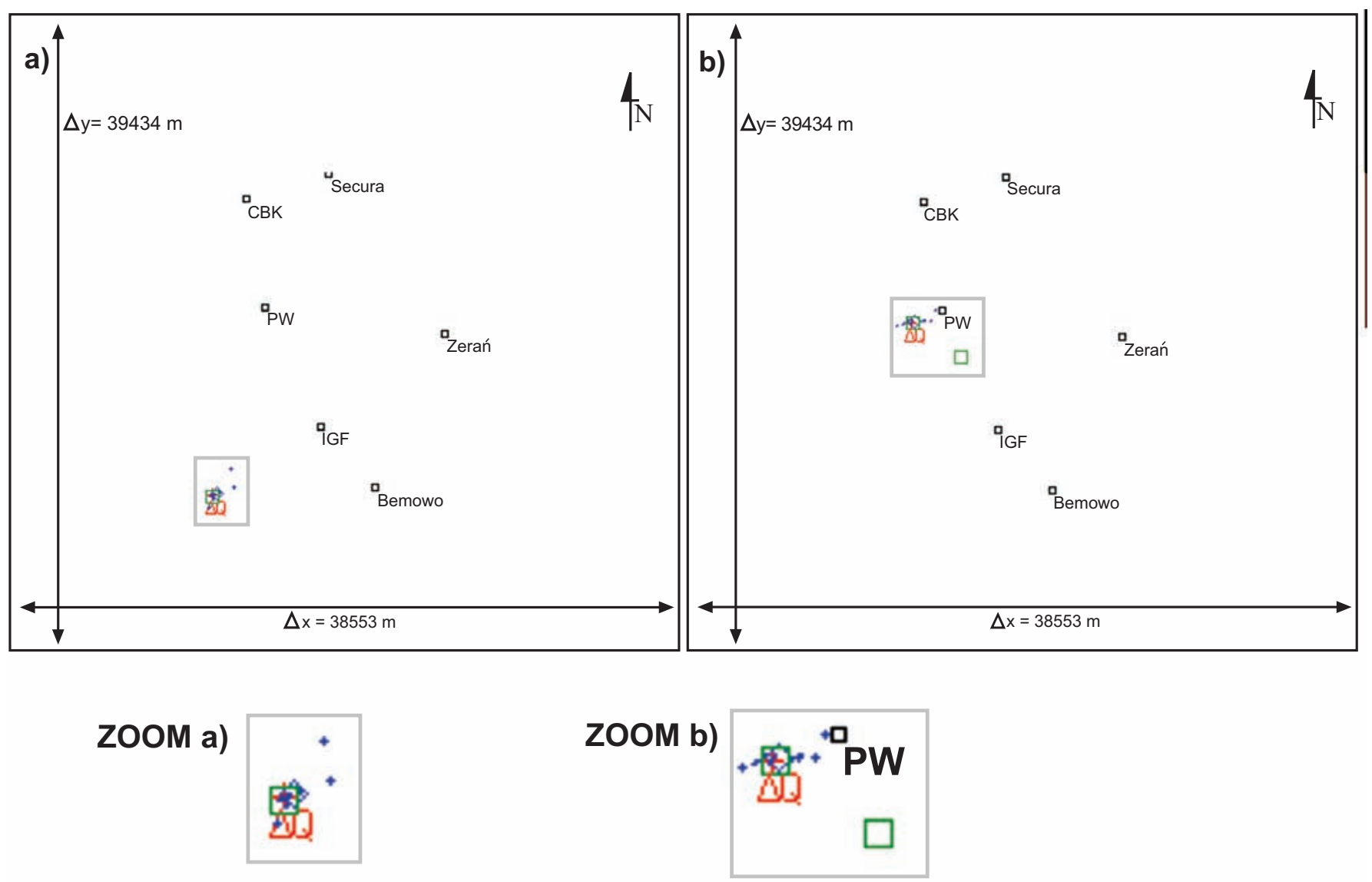

Fig. (10). Simulations for electric charge location involved in a lightning flash, obtained from the simplex method (a) and the method of linear equations $(\mathbf{b})$. 
In case (b) was used the simplex method that has not revealed the global minimum after the first calculation step, so that the locating procedure had to be started again from the another initial point; thus, location errors indicated by the repeated simplex procedure were $\delta \mathrm{x}=43 \mathrm{~m}$ and $\delta \mathrm{y}=3 \mathrm{~m}$, whereas those errors given by using the alternative method of linear equations were $\delta \mathrm{x}=-148 \mathrm{~m}$ and $\delta \mathrm{y}=-88 \mathrm{~m}$.

The indicated area is $20 \times 20 \mathrm{~km}$, the black small squares gives the position of particular LLDN stations, red cross denote the assumed electric charge position, green square shows the location of the charge obtained from the simplex method, blue diamond points represent the charge location obtained from use of the method of linear equations, and small blue crosses represent the solutions of all combinations of the four equations derived from the network of six measuring stations.

\section{CONCLUSIONS}

Although lightning location works successfully and for a long time in many countries, a number of questions remain that deserve continued investigation. The present contribution demonstrates the usefulness of comparisons between different networks operating in the same area. Especially the use of very different measuring techniques can lead to quite interesting results, and the employment of small local networks with specialized equipment helps to understand some of the important details associated with complex lightning discharge processes. For these reasons, the described comparisons will be continued in order to confirm the results already obtained and to find out whether different types of storms produce marked changes in the extracted lightning parameters. Finally, data comparisons will be extended to other areas and different networks, and increasing efforts will be undertaken in order to reconcile the experimental findings with the present theoretical models, mainly concerning discharge initiation, advent of leader steps, and development of CG- and IC-strokes.

\section{ACKNOWLEDGMENTS}

The authors acknowledge the contribution to financing of this work by the Polish Ministry of Science and Higher Edu- cation as a granted research project in 2007 - 2009. Hans D. Betz acknowledges partial support of this work by Deutsche Forschungsgemeinschaft (DFG) and Bundesministerium für Bildung und Forschung (project RegioExAKT by the Federal Ministry BMBF).

\section{REFERENCES}

[1] Betz HD, Schmidt K, Oettinger WP, Wirz M. Total VLF/VFLightning and Pseudo 3D-Discriminatuion of Intra-Cloud and Cloud-to Ground Discharges, Proc of $18^{\text {th }}$ International Lightning Detection Conference, Helsinki (Finland), 7-9 June 2004,

[2] Betz HD, Schmidt K, Fuchs B, Oettinger WP, Höller H. Cloud Lightning: Detection and Utilization for Total Lightning measured in the VLF/LF Regime. J Lightning Res 2007; 2: 1-17.

[3] Schmidt K, Oettinger WP, Betz HD, Wirz M, Diendorfer G. A New Lightning detection Network in Southern Germany, $27^{\text {th }}$ Conf. on Lightning Protection (ICLP), Avignon (France), Sept. 2004,

[4] Schmidt K, Betz HD, Oettinger WP, et al. A Comparative Analysis of Lightning Data during the EU TROCCINOX / TroCCiBras Campaign, VIII ${ }^{\text {th }}$ International Symposium on Lightning Protection, Sao Paulo, Brazil, $21^{\text {st }}-25^{\text {th }}$ November 2005.

[5] Loboda M, Maslowski G, Dziewit Z, et al. A new Lightning Detection Network in Poland, Int Conf on Grounding and Earthing, Maceió, Brazil, 2006.

[6] Betz HD, Schmidt K, Fuchs B, Oettinger WP, Höller H. Cloud lightning: detection and utilization for total lightning measured in the VLF/LF regime. J Lightning Res 2007; 2: 1-17.

[7] Betz HD, Marshall TC, Stolzenburg M, et al. Detection of in-cloud lightning with VLF/LF and VHF networks for studies of the initial discharge phase. Geophys Res Lett 2008; 2008GL035820.

[8] Betz HD, Schmidt K, Oettinger WP. LINET - An international VLF/LF lightning detection network in Europe, in: Lightning: principles, instruments and applications, Betz HD, Schumann U, Laroche P, Eds. ch 5, Dordrecht (NL), Springer, 2008.

[9] Richard P, Lojou JY. Assessment of application of storm cell electrical activity monitoring to intense precipitation forecast. $10^{\text {th }}$ Int. Conf. on Atmos Electricity, Osaka, Japan, 1996; p. 284-287.

[10] NIMA Technical Report TR8350.2, Department of Defense, World Geodetic System 1984, Its Definition and Relationships With Local Geodetic Systems. $3^{\text {rd }}$ ed, Geospatial-Intelligence Agency, 1997.

[11] Krehbiel PR, Brook M, McCrory RA. An Analysis of the Charge Structure of Lighting Discharges to Ground. J Geophys Res 1979; 84: p. 2432-2456.

[12] Jacobson EA, Krider EP. Electrostatic Field Changes Produced by Florida Lighting. J Atmos Sci 1976; 33: pp. 103-117.

[13] Debski W. Application of Monte Carlo Techniques for Solving Selected Seismological Inverse Problems. Publs Inst Geophys Pol Acad Sc 2004; B-34 (367): 32-57. 